\title{
Does social influence affect COVID-19 vaccination intention among the unvaccinated?
}

Gul Deniz Salali ${ }^{*}$, Mete Sefa Uysal ${ }^{2}$, Gizem Bozyel ${ }^{3}$, Ege Akpinar ${ }^{4}$, Ayca Aksu ${ }^{5}$

${ }^{1}$ Department of Anthropology, University College London, 14 Taviton Street, WC1H OBW, United Kingdom

2Department of Social Psychology, Friedrich Schiller University Jena, Jena, Germany ${ }^{3}$ Department of Psychology, Dokuz Eylul University, Izmir, Turkey

${ }^{4}$ Deparment of Political Science and International Relations, Altinbas University, Istanbul, Turkey

${ }^{5}$ Department of Psychology, MEF University, Istanbul, Turkey

*Correspondence: guldeniz.salali@ucl.ac.uk

Keywords: conformism, vaccine hesitancy, psychological reactance, collectivism, cultural evolution 


\section{Abstract}

Conformist social influence is a double-edged sword when it comes to vaccine promotion. On the one hand, social influence may increase vaccine uptake by reassuring the hesitant about the safety and effectiveness of the vaccine; on the other, people may forgo the cost of vaccination when the majority is already vaccinated giving rise to a public goods dilemma. Here, we examine whether available information on the percentage of double-vaccinated people affects COVID-19 vaccination intention among unvaccinated people in Turkey. In an online experiment, we divided participants $(n=1013)$ into low, intermediate, and high social influence conditions, reflecting the government's vaccine promotion messages. We found that social influence did not predict COVID-19 vaccination intention, but psychological reactance and collectivism did. People with higher reactance (intolerance of others telling one what to do and being sceptical of consensus views) had lower vaccination intention, whilst people with higher collectivism (how much a person considers group benefits over individual success) had higher vaccination intention. Our findings suggest that advertising the percentage of double-vaccinated people is not sufficient to trigger a cascade of others getting themselves vaccinated. Diverse promotion strategies reflecting the heterogeneity of individual attitudes could be more effective.

\section{Introduction}

Vaccination is a social dilemma. The more people are vaccinated, the better protected the group is against infectious diseases; however, not everyone is willing to get vaccinated. As vaccination programs proceed, they can stall because the remaining unvaccinated mainly consists of those who strongly hesitate or refuse to get the vaccine. Ongoing vaccination campaigns provide a natural setting for understanding what strategies work best for increasing vaccine uptake among unvaccinated people. Governments use various strategies to increase vaccination coverage. One of those strategies is using social influence by revealing how many people in the population have received the vaccine and encouraging others to follow. In this study, we tested the effectiveness of this strategy in increasing vaccination intention among the unvaccinated using an online experiment in Turkey.

We focused on Turkey for several reasons. First, our previous surveys revealed that general and COVID-19 vaccine hesitancy is high and trust in vaccines low in Turkey compared to other countries (Salali \& Uysal 2020, 2021b). Second, conformist social influence has been actively used as a COVID-19 vaccine promotion strategy in this country. Since late September 2021, the percentage of people who have received at least two doses of the COVID-19 vaccine in each city has been announced daily by the Turkish Health Minister on a colour-coded map on social media, television, and the Internet (Figure 1). These announcements included plaudits for the cities that reached over $75 \%$ coverage and encouraged others to follow. Third, at the time of our 
experiment, over $65 \%$ of the adult population in Turkey had received the two doses of the COVID-19 vaccine, and the remaining unvaccinated adults in big cities mostly consisted of those who did not get the vaccine despite availability.

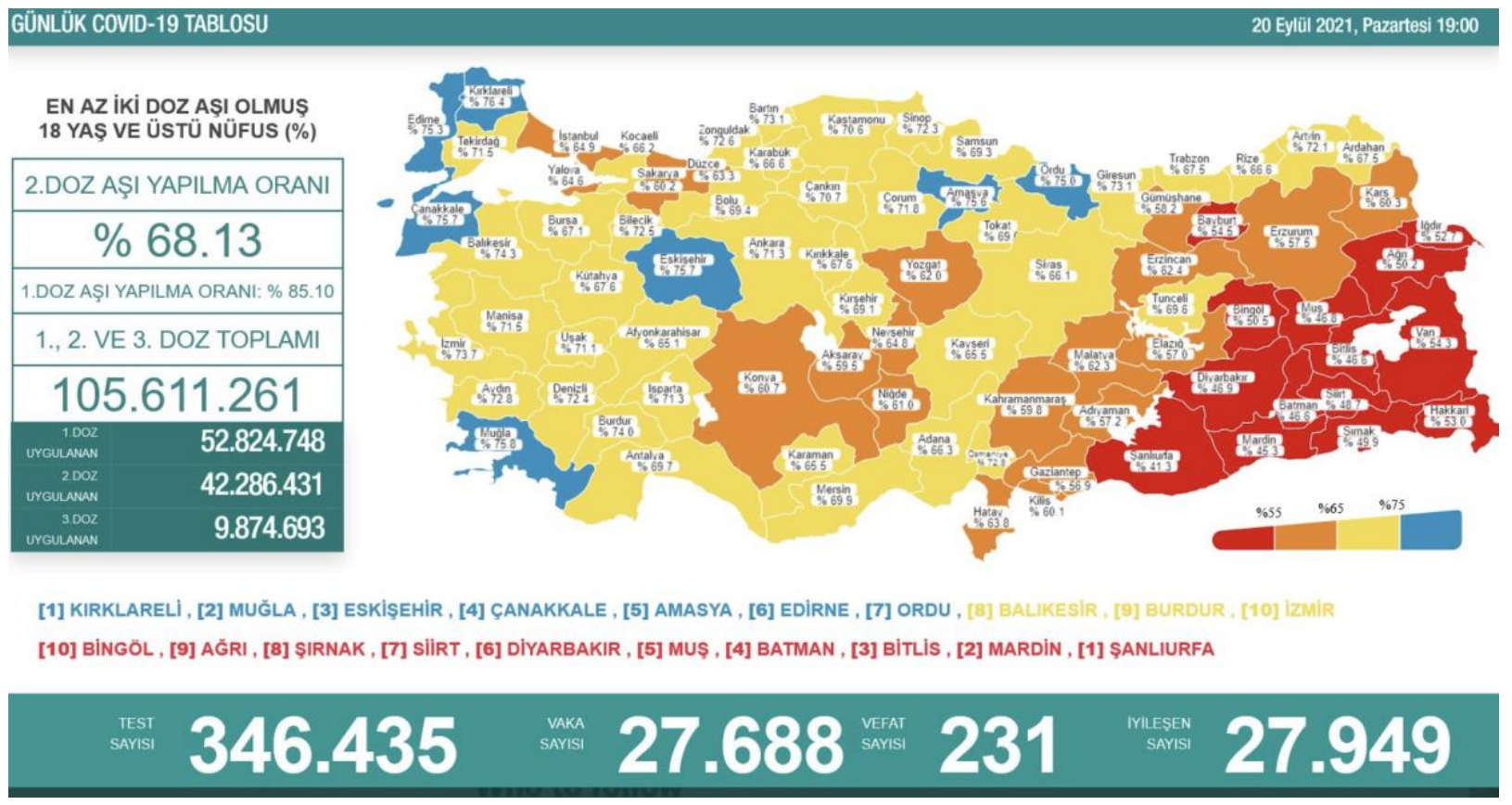

Figure 1. The infographic used by the Turkish Ministry of Health illustrating the percentage of double-vaccinated people in each city at the time of this study in late September 2021. Blue indicates cities where over $75 \%$ of the adults have received the two doses of the COVID-19 vaccine, yellow indicates over $65 \%$, orange over $55 \%$ and red under $55 \%$. (Source: https://covid19asi.saglik.gov.tr)

\section{Conformist social influence and the vaccination dilemma}

Conformism refers to the tendency of people to learn from and adopt the behaviours of the majority in their group (Asch 1956). Evolutionary human scientists have argued that conformism is adaptive unless environments change too rapidly or individual learning is error free (Boyd \& Richerson 1985; Henrich \& Boyd 1998). Conformist social influence may contribute to the spread of cultural practices with health consequences such as female genital cutting (Efferson et al. 2020; Howard \& Gibson 2017 ) and the adoption of new health technologies. A new vaccine constitutes a prime example. The novelty raises questions about unforeseen side effects and efficiency of the vaccine and may lead to hesitancy, especially when the origin of the disease is largely unknown (Salali \& Uysal 2020). Seeing others getting vaccinated may therefore encourage hesitant individuals, especially if they have concerns about vaccine side effects. Through a cross-cultural survey, we found that vaccination of friends and family were amongst the most effective incentives in increasing vaccination intention among participants who were hesitant about getting the COVID19 vaccine, especially in Turkey (Salali \& Uysal 2021a). Likewise, a recent theoretical model predicted that willingness to be vaccinated against COVID-19 may increase as 
more and more people get vaccinated (Schmelz \& Bowles 2021). Because conformist social influence depends on the number of people in a community adopting the behaviour, we expect there to be a threshold percentage of people who already got the vaccine for the vaccination behaviour to take off in a group.

On the other hand, achieving herd immunity through voluntary vaccination represents a public goods dilemma (Bauch \& Earn 2004). People may adopt a "cheater" or "freerider" strategy to avoid the (perceived) cost of getting vaccinated if most in their community have already received the vaccine (lbuka et al. 2014). This is especially true for individuals who perceive the cost of vaccination (e.g. unforeseen side effects) to be high (Bauch \& Earn 2004; Fu et al. 2011). The temptation to free ride may result in lowered uptake of vaccinations as the percentage of the vaccinated increases. This is because, at higher levels of vaccination coverage, there will be a lower risk of infection for both the vaccinated and the unvaccinated. This scenario represents a classic example of the public goods dilemma (Hardin 1968) and is likely to be responsible for the observed decline in measles vaccine coverage across Europe in the last few decades (Jansen et al. 2003; WHO 2020).

Based on the above, we predict that the effect of social influence on vaccine uptake will follow a reverse U-shaped pattern: at lower percentages of vaccinations in a population, there will not be enough consensus for conformity to kick off. At intermediate levels of vaccination coverage, conformist social influence will amplify vaccine uptake. At higher percentages, however, the unvaccinated will become disincentivised from vaccinating as they benefit from the growing herd immunity (Figure 2).

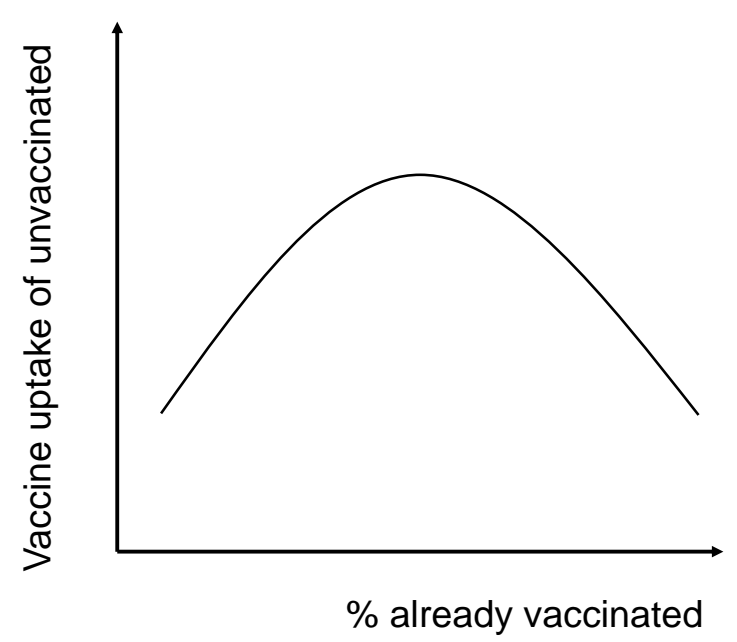

Figure 2. Predicted effect of social influence on the vaccine uptake of unvaccinated people. At lower percentages of vaccine coverage, there will not be enough people for conformist social influence to have an effect. At intermediate percentages, conformist influence will contribute to the increase in vaccination behaviour. At higher percentages, the remaining unvaccinated may free ride on the benefits of herd protection. 
There is another factor that should be considered when examining the effect of social influence on vaccination intention: the susceptibility of an individual to be influenced by the majority. Psychological attitudes contribute to this susceptibility. For example, individuals who are highly in need of uniqueness are found to resist majority influence (Imhoff \& Erb 2009). Likewise, psychological reactance refers to the defensive response to and intolerance of others telling one how to think (Brehm 1966) and is negatively correlated with tendency to conform (Goldsmith et al. 2005). Someone high in reactance perceives advice from others and compliance to social norms as an intrusion on one's freedom and autonomy. Studies have revealed positive links between psychological reactance, belief in conspiracies, and vaccine hesitancy (Hornsey et al. 2018) (Salali et al. unpublished observations) and refusing to wear a facemask during the COVID-19 pandemic (Taylor \& Asmundson 2021). It is possible that psychologically reactant people signal their nonconformity by refusing the COVID19 vaccine.

Collectivism (the extent to which an individual considers group welfare and loyalty over individual success) may also play a role in vaccine uptake and the degree of the effect of conformist social influence on vaccine uptake. First, a highly collectivistic individual may accept vaccination more readily as they value the benefit of herd immunity. Related research has found that mask usage during the COVID-19 pandemic was higher in more collectivistic US states and countries (Lu et al. 2021). People in more collectivistic (versus individualistic) cultures may also be more likely to accept various contact-tracing technologies during a pandemic (Arnot et al. 2020). Second, conformist social influence may have a larger influence on collectivistic individuals as they are likely to be more attuned to how people in their group behave. People in more collectivistic cultures, for example, are more likely to conform to majority opinion (Bond \& Smith 1996). Likewise, computational models have revealed that majority opinion solidifies more quickly in collectivistic cultures where there is greater susceptibility to social influence (Muthukrishna \& Schaller 2020).

Most studies to date have used game theoretical models (Bauch \& Earn 2004; Fu et al. 2011; Schmelz \& Bowles 2021) or hypothetical disease scenarios (lbuka et al. 2014) to investigate how social information influences vaccination behaviour. This study uniquely contributes by empirically assessing the role of social influence on vaccination intention among the unvaccinated during the COVID-19 pandemic. It also fills a gap in the literature by examining how psychological factors may mitigate the effect of conformist social influence on the adoption of a new health behaviour. Our study tested the following predictions via an online experiment in Turkey with participants who had not yet been vaccinated against COVID-19: 
1) Vaccination intention of unvaccinated individuals will be low at low levels of vaccine coverage, increase with increasing levels, and decrease past intermediate levels due to the vaccination dilemma (Figure 1).

2) Unvaccinated people who score higher on the psychological reactance scale will have lower intention to get the COVID-19 vaccine.

3) Unvaccinated people who score higher on collectivism will have higher intention to get the COVID-19 vaccine.

4) The association with conformist social influence and vaccination intention will be moderated by psychological reactance and collectivism. More specifically, we predict that the positive influence of conformism at intermediate levels of vaccine coverage will not hold for people who score high on reactance. On the other hand, we predict social influence to have a higher effect on people who score higher on collectivism.

\section{Methods}

We conducted an anonymous online experiment with a sample of participants in Turkey from 29 September to 8 October 2021. We used Qualtrics, which is a commercial survey administration company. Qualtrics recruited participants from their pool of potential participants who have agreed to be contacted for the purpose of responding to surveys. Our inclusion criterion was that the participant did not receive any doses of the COVID-19 vaccine (we excluded those who replied "yes" to our initial check question regarding whether they received at least one dose of the vaccine). We used filters to eliminate data from careless or incomplete responses.

In the first part of the online experiment, we gathered information about the psychological and control variables used in the study. Then, we randomly divided the participants into control and experimental conditions and asked manipulation check questions. At the end of the online study, we asked participants about the probability that they would receive the COVID-19 vaccine. The following sections detail each component of the study.

\section{Psychological and control variables}

We measured psychological reactance using the reactance scale (Hong \& Faedda 1996) and collectivism using the collectivism-individualism scale (Hofstede 2001). As native speakers, we translated all scale questions into Turkish and back-translated them for accuracy. We also gathered information on several demographic variables, including the cities and districts in which the participants were living, as well as sex, age, education level, income, and political orientation. We asked about districts to ensure that the participants trusted the information we provided in the experimental condition. Table 1 presents the psychological and demographic variables that were measured in the experiment, the statements employed to capture the variables, the response scales, Cronbach's alphas, and means or percentages. 
Table 1. Summary of the sample and variables

\begin{tabular}{|c|c|c|}
\hline Variable & Statement(s) & Mean (SD) / Frequency \\
\hline \multirow{4}{*}{$\begin{array}{l}\text { Vaccine Intention } \\
\text { (Outcome variable) }\end{array}$} & What is your probability of & $0-25: 31.3 \%$ \\
\hline & getting a COVID-19 & $26-50: 23.4 \%$ \\
\hline & vaccine? (0: Definitely will & 51-75: $19.8 \%$ \\
\hline & not- 100: Definitely will) & $76-100: 25.5 \%$ \\
\hline \multirow[t]{6}{*}{$\begin{array}{l}\text { Psychological Reactance (a } \\
=.67)\end{array}$} & $\begin{array}{l}(1=\text { completely disagree, } 5= \\
\text { completely agree })\end{array}$ & $3.59(.70)$ \\
\hline & $\begin{array}{l}\text { 1. I become angry when my } \\
\text { freedom of choice is } \\
\text { restricted. }\end{array}$ & \\
\hline & $\begin{array}{l}\text { 2. I find contradicting others } \\
\text { stimulating. }\end{array}$ & \\
\hline & $\begin{array}{l}\text { 3. It disappoints me to see } \\
\text { others submitting to } \\
\text { society's standards and } \\
\text { rules. }\end{array}$ & \\
\hline & $\begin{array}{l}\text { 4. I resist the attempts of } \\
\text { others to influence me. }\end{array}$ & \\
\hline & $\begin{array}{l}\text { 5. I consider advice from } \\
\text { others to be an intrusion. }\end{array}$ & \\
\hline \multirow[t]{7}{*}{$\begin{array}{l}\text { Collectivism } \\
(a=.89)\end{array}$} & $\begin{array}{l}\text { (1= completely disagree, } 5= \\
\text { completely agree })\end{array}$ & $3.32(.88)$ \\
\hline & $\begin{array}{l}\text { 1. Individuals should } \\
\text { sacrifice self-interest for } \\
\text { the group. }\end{array}$ & \\
\hline & $\begin{array}{l}\text { 2. Individuals should stick } \\
\text { with the group even } \\
\text { through difficulties. }\end{array}$ & \\
\hline & $\begin{array}{l}\text { 3. Group welfare is more } \\
\text { important than individual } \\
\text { rewards. }\end{array}$ & \\
\hline & $\begin{array}{l}\text { 4. Group success is more } \\
\text { important than individual } \\
\text { success. }\end{array}$ & \\
\hline & $\begin{array}{l}\text { 5. Individuals should only } \\
\text { pursue their goals after } \\
\text { considering the welfare } \\
\text { of the group. }\end{array}$ & \\
\hline & $\begin{array}{l}\text { 6. Group loyalty should be } \\
\text { encouraged even if } \\
\text { individual goals suffer. }\end{array}$ & \\
\hline Age & & $35.95(9.96)$ \\
\hline Gender & Women & $67.2 \%$ \\
\hline
\end{tabular}




\begin{tabular}{llc} 
& Men & $32.7 \%$ \\
& Non-binary/Other & $0.1 \%$ \\
\hline Education Level & Elementary School & $1.2 \%$ \\
& Middle School & $3.1 \%$ \\
& High School & $23.9 \%$ \\
& Bachelor's Degree & $51.3 \%$ \\
& Master's Degree & $18.1 \%$ \\
& PhD & $2.4 \%$ \\
\hline Income Level & Less than 2.500 Turkish & $10.1 \%$ \\
& Liras (TL) & \\
& $2.500-4.999$ TL & $21.1 \%$ \\
& $5.000-7.499$ TL & $20.8 \%$ \\
& $7.500-9.999$ TL & $25.8 \%$ \\
& More than 10.000 TL & $22.2 \%$ \\
\hline City & Istanbul - Ankara - İzmir & $64.6 \%$ \\
& Other cities & $35.4 \%$ \\
\hline Political Orientation & Left (0-4) & $25.7 \%$ \\
& Center (5-6) & $45.1 \%$ \\
& Right (7-10) & $29.2 \%$ \\
\hline
\end{tabular}

\section{Experimental conditions}

To test our first prediction related to the conformist social influence on vaccination intention, we randomly divided the participants into four experimental conditions. Participants received the following information in each condition:

"On the next page, you will receive some information. Please read this information carefully before continuing the survey."

Control condition: "As you know, there has been an ongoing vaccine rollout against the COVID-19 pandemic in our country."

Low social influence condition ( $30 \%$ vaccinated): "As you know, there has been an ongoing vaccine rollout against the COVID-19 pandemic in our country. As part of this rollout, $30 \%$ of the people in the district that you are living in have gotten their two doses of the COVID-19 vaccine."

Intermediate social influence condition (60\% vaccinated): "As you know, there has been an ongoing vaccine rollout against the COVID-19 pandemic in our country. As part of this rollout, $60 \%$ of the people in the district that you are living in have gotten their two doses of the COVID-19 vaccine." 
High social influence condition ( $90 \%$ vaccinated): "As you know, there has been an ongoing vaccine rollout against the COVID-19 pandemic in our country. As part of this rollout, $90 \%$ of the people in the district that you are living in have gotten their two doses of the COVID-19 vaccine."

Participants had to wait 7 seconds before moving onto the next page to ensure that they read the information.

\section{Manipulation check}

To check whether our experimental conditions worked, we asked participants to rate the level of double vaccinations in their district after they received the information assigned to their condition. Specifically, we asked, "What do you think about the proportion of people in your district who received the two doses of the COVID-19 vaccine?" We prompted the participants to choose between the following options: low, intermediate, and high. If the experimental manipulation worked, we expected participants in the low social influence condition to choose the "low" option more than those in the control and other conditions. Likewise, we expected participants in the high social influence condition to choose the "high" option more often than those in other conditions. We coded the responses on a scale of 1 to $3(1=$ low, $2=$ intermediate, $3=$ high) and compared the responses in the control condition with the others using ANOVA. As predicted, participants in the low social influence condition reported significantly lower scores than those in the control condition $\left(M_{\text {control }}=2.04\right.$, $\mathrm{SD}=.60 ;$ Mexperiment-low $=1.81, \mathrm{SD}=.66 ; 95 \%[.09, .39])$. Responses in the control and intermediate conditions did not differ $\left(\mathrm{M}_{\text {control }}=2.04, \mathrm{SD}=.60\right.$; Mexperiment-intermediate $=$ $2.12, \mathrm{SD}=.57 ; 95 \%[-.22, .07])$. Those in the high social influence condition reported significantly higher scores than those in the control condition $\left(M_{\text {control }}=2.04, S D=.60\right.$; Mexperiment-high $=2.25, \mathrm{SD}=.70 ; 95 \%[-.36,-.07])$. Thus, we concluded that our manipulations had worked as expected.

Outcome variable: At the end of the online experiment, each participant was asked to rate their probability of vaccination against COVID-19 on a scale of 0 to 100 (see Table 1).

\section{Statistical analyses}

We conducted quasibinomial logistic regression analyses in $\mathrm{R}$ to test the prediction power of 1) social influence conditions (control/30\%/60\%/90\% double vaccinated), 2) psychological reactance score, 3) collectivism score, and 4) demographic controls (age, sex, education level, income, political orientation) on COVID-19 vaccination intention ( 0 [no intention] - 1 [full intention]). We used ANOVA to compare the regression models. 


\section{Results}

\section{Social influence did not predict vaccination intention among unvaccinated people}

As predicted, the mean vaccination intention was highest among the participants in the intermediate $(60 \%$ vaccinated) social influence condition (Figure 3$)$. However, contrary to our first prediction, there was no significant difference in the odds of COVID-19 vaccination intention between the control and social influence conditions (Table 2, Model 1).

Table 2. Logistic regression models of the odds of COVID-19 vaccination intention among the unvaccinated in Turkey

\begin{tabular}{|c|c|c|c|c|c|c|c|c|}
\hline & Model 1 & & Model 2 & & Model 3 & & Model 4 & \\
\hline & $\begin{array}{l}\text { Odds Ratio } \\
\text { (Confidence } \\
\text { Intervals) }\end{array}$ & $\begin{array}{l}p \\
\text { value }\end{array}$ & $\begin{array}{l}\text { Odds Ratio } \\
\text { (Confidence } \\
\text { Intervals) }\end{array}$ & $\begin{array}{l}p \\
\text { value }\end{array}$ & $\begin{array}{l}\text { Odds Ratio } \\
\text { (Confidence } \\
\text { Intervals) }\end{array}$ & $\begin{array}{l}p \\
\text { value }\end{array}$ & $\begin{array}{l}\text { Odds Ratio } \\
\text { (Confidence } \\
\text { Intervals) }\end{array}$ & $\begin{array}{l}p \\
\text { value }\end{array}$ \\
\hline $\begin{array}{l}\text { Low social } \\
\text { influence: \%30 } \\
\text { vaccinated }\end{array}$ & $\begin{array}{c}.97 \\
(0.77,1.23)\end{array}$ & 0.824 & & & $\begin{array}{c}0.97 \\
(0.76,1.23)\end{array}$ & 0.790 & $\begin{array}{c}0.34 \\
(0.09,1.35)\end{array}$ & 0.125 \\
\hline $\begin{array}{l}\text { Intermediate } \\
\text { social } \\
\text { influence: \%60 } \\
\text { vaccinated }\end{array}$ & $\begin{array}{c}1.13 \\
(0.90,1.43)\end{array}$ & 0.293 & & & $\begin{array}{c}1.14 \\
(0.90,1.44)\end{array}$ & 0.270 & $\begin{array}{c}0.48 \\
(0.12,1.96)\end{array}$ & 0.310 \\
\hline $\begin{array}{l}\text { High social } \\
\text { influence: \%90 } \\
\text { vaccinated }\end{array}$ & $\begin{array}{c}0.94 \\
(0.74,1.18)\end{array}$ & 0.579 & & & $\begin{array}{c}0.94 \\
(0.74,1.18)\end{array}$ & 0.580 & $\begin{array}{c}0.90 \\
(0.23,3.51)\end{array}$ & 0.877 \\
\hline $\begin{array}{l}\text { Psychological } \\
\text { Reactance }\end{array}$ & & & $\begin{array}{c}0.80 \\
(0.71,0.91)\end{array}$ & .001 & $\begin{array}{c}0.80 \\
(0.70,0.91)\end{array}$ & $\begin{array}{c}< \\
.001\end{array}$ & $\begin{array}{c}0.81 \\
(0.64,1.03)\end{array}$ & 0.083 \\
\hline Collectivism & & & $\begin{array}{c}1.14 \\
(1.04,1.27)\end{array}$ & .009 & $\begin{array}{c}1.14 \\
(1.04,1.27)\end{array}$ & .010 & $\begin{array}{c}0.98 \\
(0.81,1.19)\end{array}$ & 0.833 \\
\hline $\begin{array}{l}\text { Gender } \quad(0= \\
\text { Women, } \quad 1= \\
\text { Men })\end{array}$ & $\begin{array}{c}0.78 \\
(0.65,0.94)\end{array}$ & 0.009 & $\begin{array}{c}0.78 \\
(0.65,0.94)\end{array}$ & .010 & $\begin{array}{c}0.78 \\
(0.65,0.94)\end{array}$ & 0.010 & $\begin{array}{c}0.77 \\
(0.64,0.93)\end{array}$ & 0.010 \\
\hline Age & $\begin{array}{c}1.00 \\
(0.99,1.01)\end{array}$ & 0.462 & $\begin{array}{c}1.00 \\
(0.99,1.01)\end{array}$ & 0.861 & $\begin{array}{c}1.00 \\
(0.99,1.01)\end{array}$ & 0.900 & $\begin{array}{c}1.00 \\
(0.99,1.01)\end{array}$ & 0.914 \\
\hline Education & $\begin{array}{c}0.93 \\
(0.84,1.04)\end{array}$ & 0.189 & $\begin{array}{c}0.95 \\
(0.86,1.06)\end{array}$ & 0.383 & $\begin{array}{c}0.96 \\
(0.86,1.06)\end{array}$ & 0.410 & $\begin{array}{c}0.96 \\
(0.86,1.07)\end{array}$ & 0.418 \\
\hline Income & $\begin{array}{c}1.06 \\
(0.98,1.14)\end{array}$ & 0.166 & $\begin{array}{c}1.07 \\
(0.99,1.15)\end{array}$ & 0.095 & $\begin{array}{c}1.07 \\
(0.99,1.16)\end{array}$ & 0.080 & $\begin{array}{c}1.07 \\
(0.99,1.16)\end{array}$ & 0.081 \\
\hline $\begin{array}{l}\text { Political } \\
\text { Orientation }(0 \\
=\text { Left, } 10= \\
\text { Right })\end{array}$ & $\begin{array}{c}1.08 \\
(1.04,1.11)\end{array}$ & $\begin{array}{c}< \\
.001\end{array}$ & $\begin{array}{c}1.07 \\
(1.03,1.10)\end{array}$ & $\begin{array}{c}< \\
.001\end{array}$ & $\begin{array}{c}1.07 \\
(1.03,1.10)\end{array}$ & $\begin{array}{c}< \\
.001\end{array}$ & $\begin{array}{c}1.07 \\
(1.03,1.10)\end{array}$ & $\begin{array}{c}< \\
.001\end{array}$ \\
\hline $\begin{array}{l}\text { Reactance } x \\
\text { low social } \\
\text { influence }\end{array}$ & & & & & & & $\begin{array}{c}1.11 \\
(0.78,1.57)\end{array}$ & .560 \\
\hline $\begin{array}{l}\text { Reactance } \mathrm{x} \\
\text { intermediate }\end{array}$ & & & & & & & $\begin{array}{c}0.93 \\
(0.66,1.32)\end{array}$ & .695 \\
\hline
\end{tabular}


social

influence

Reactance $\mathrm{x}$

0.92

.613

high social

$(0.65,1.29)$

influence

Collectivism $\mathrm{x}$

1.22

0.161

low social

$(0.92,1.62)$

influence

Collectivism $\mathrm{x}$

1.39

0.020

intermediate

$(1.06,185)$

social

influence

Collectivism $\mathrm{x}$

1.11

0.432

high social

influence

$(0.85,1.46)$

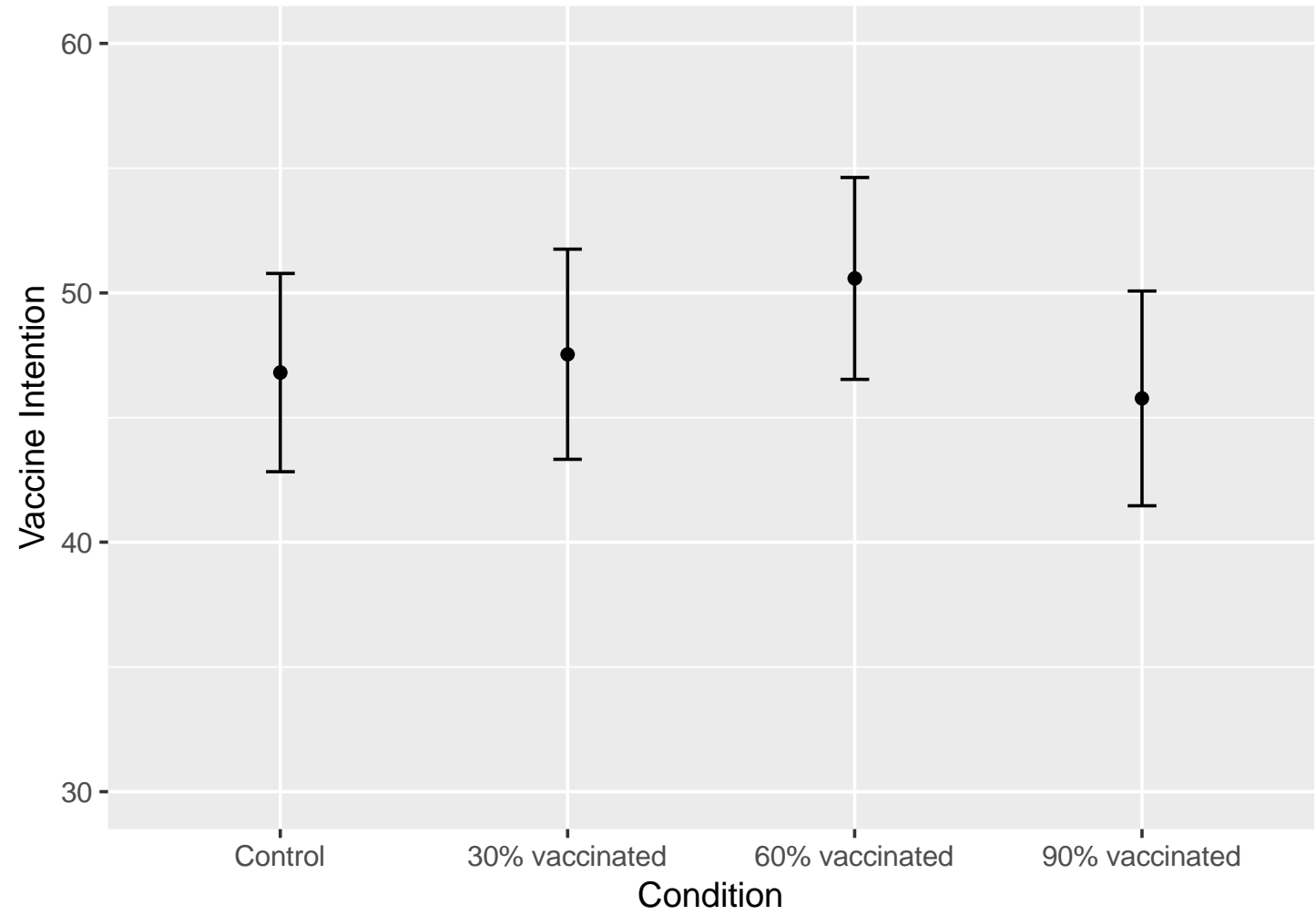

Figure 3. Mean COVID-19 vaccination intention among the unvaccinated in Turkey by experimental conditions (control: $n=263$; social influence: $30 \%$ double vaccinated $n=248$, $60 \%$ double vaccinated $n=254,90 \%$ double vaccinated $n=270)$. Error bars indicate $95 \%$ confidence intervals.

Collectivistic unvaccinated had higher, reactant unvaccinated had lower vaccination intention 
In line with Predictions 2 and 3 , both collectivism and psychological reactance scores predicted the odds of vaccination intention among the unvaccinated (Figure 4). The odds of COVID-19 vaccination intention increased by $14 \%$ with every one-point increase in the mean collectivism score (Table 2, Model 2). In contrast, a point increase in the psychological reactance score was associated with a $25 \%$ decrease in the odds of vaccination intention (Table 2, Model 2).

One of our predictions (\#4) was that the susceptibility to social influence would be moderated by psychological reactance and collectivism. We expected people who scored higher on psychological reactance to be less influenced by others' vaccination behaviour. Nevertheless, the reactance $x$ social influence interaction terms were nonsignificant predictors of vaccination intention (Table 2, Model 4). To further test whether social influence changed the odds of vaccination intention among the participants who scored low on reactance, we performed an additional regression analysis with the subgroup of participants whose reactance mean score was less than three. Social influence did not predict vaccination intention in this subgroup either ( $30 \%$ condition, $p=0.78 ; 60 \%$ condition, $p=0.95 ; 90 \%$ condition, $p=0.42$ ).

As predicted, there was a positive collectivism $x$ social influence interaction at the intermediate (60\%) level (Table 2, Model 4). In other words, unvaccinated people who scored higher on collectivism and who received the information that $60 \%$ of those in their district were double vaccinated had the highest vaccination intention. Nevertheless, our model testing revealed that the models in which we included experimental conditions (social influence), psychological variables, and demographic controls did not differ from the model that only included psychological variables and demographic controls (Table 2, Models 2 and 3, $F(3,1001)=1.04, p=0.37$; Models 2 and $4, F(9,995)=1.20, p=0.29)$. Therefore, the minimal adequate model was the one which did not include social influence but only included psychological variables (Table 2, Model 2). 


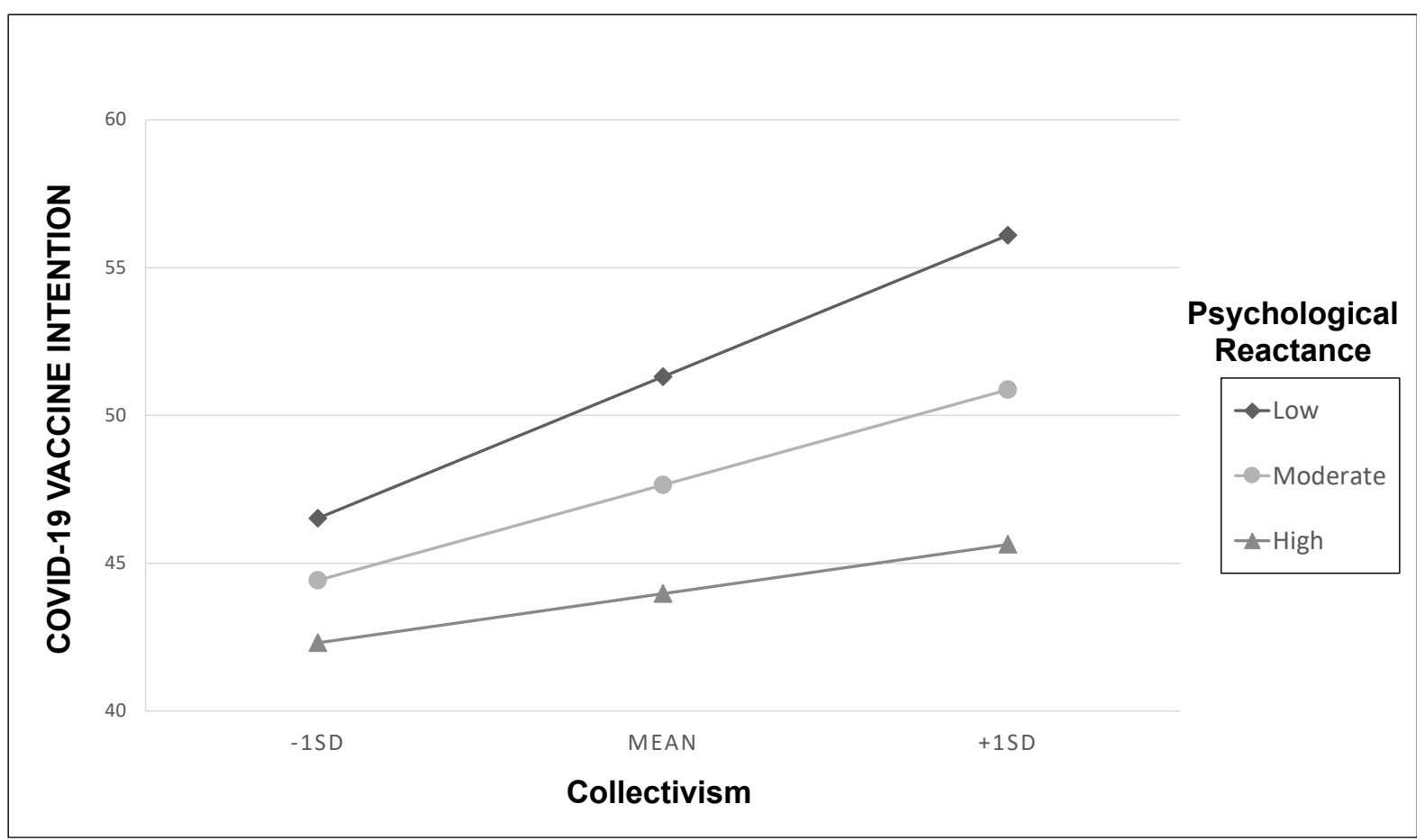

Figure 4. Vaccination intention among unvaccinated people in Turkey by their collectivism and psychological reactance scores.

Among our control variables, we found that unvaccinated men were $28 \%$ less likely to get the COVID-19 vaccine than unvaccinated women (Table 2, Model 2). Moreover, a one-point increase from left- to right-wing political orientation was associated with a $7 \%$ increase in the odds of vaccination intention (Table 2, Model 2). Age, income, and education were not significant predictors of COVID-19 vaccination intention among the unvaccinated (Table 2, Model 2).

\section{Discussion}

In this paper, we examined whether using the potential of conformist social influence is an effective vaccine promotion strategy. We seized the opportunity of the ongoing COVID-19 vaccine rollout to design a realistic study and captured the attitudes of the remaining unvaccinated. We found that information on the percentage of doublevaccinated people did not influence the odds of COVID-19 vaccination among unvaccinated people in Turkey. Two psychological variables, however, predicted their vaccination intention. People who scored higher on collectivism had higher vaccination intention, and people who scored higher on psychological reactance had lower vaccination intention. Our findings also indicate that unvaccinated women and those with a right-wing political orientation had higher odds of COVID-19 vaccination.

Using computational models, researchers have demonstrated that the willingness to be vaccinated may increase over time in response to the percentage of the population already vaccinated and argue that the media should focus on reporting those already 
vaccinated to induce a cascade of vaccinations (Schmelz \& Bowles 2021). Here, we took this a step further and predicted that while this conformist social influence may work at intermediate percentages of vaccination coverage, at high percentages of coverage, social influence may cease to work due to vaccination becoming a public goods dilemma. Contrary to our prediction, we found no effect of conformist social influence on COVID-19 vaccination intention. It is possible that the available information on the percentage of double-vaccinated people in one's district is not as influential as observing people within one's immediate social network getting vaccinated. For example, in a previous study, we discovered that one of the most effective incentives at increasing the reported COVID-19 vaccination intention among vaccine-hesitant people in the UK, US, and Turkey was the vaccination of friends and family members (Salali \& Uysal 2021a). Moreover, unlike other social coordination problems like mask wearing, vaccination is not a visible trait. Not everyone follows the statistics on vaccination, and one's vaccination status is not visible like a mask. This may also impact social influence.

Another possibility is that conformist social influence is not as straightforward as many policymakers hope and there is heterogeneity in individual susceptibility to social influence (Efferson et al. 2020). Indeed, our findings suggest that individual-level psychological traits are key. The extent to which a person viewed others' attempts at persuasion as an intrusion on their freedom (reactance) played a larger role in vaccination intention among the remaining unvaccinated than did social influence. This finding on reactance is inconsistent with the previous finding that antivaccination attitudes across cultures are strongest among highly reactant people (Hornsey et al. 2018). In a recent survey study, we found that psychological reactance was higher among Turkish participants than participants in the UK and the US (Salali et al. unpublished observations). We also found that reactance predicted general conspiracy mentality and belief in COVID-19 conspiracies, which, in turn, mediated the relationship between reactance and vaccine hesitancy (Salali et al. unpublished observations). One aspect of conspiracy beliefs is that they offer alternative explanations to the majority opinion and fulfil believers' need for uniqueness (Imhoff \& Lamberty 2017). It is no surprise that an individual's need for uniqueness can undermine social influence and result in nonconformity (Imhoff \& Erb 2009). The positive links between conspiracy beliefs and the pursuit of uniqueness and conspiracy beliefs and reactance suggest that highly reactant people may refuse to get vaccinated (especially when there is a large vaccine campaign) as a statement of their nonconformity.

While reactant unvaccinated people had lower odds of COVID-19 vaccination, the odds were higher among the more collectivistic unvaccinated. It is possible that people who attach importance to their group's wellbeing consider vaccination even though they hesitate. Moreover, since majority influence is predicted to have a larger effect in collectivistic cultures (Muthukrishna \& Schaller 2020), we expected unvaccinated people high in collectivism to be more affected by social influence. Indeed, we found 
that at intermediate levels of social influence ( $60 \%$ vaccinated), more collectivistic participants indicated higher vaccination intention. Nevertheless, collectivism and reactance alone, regardless of social influence, were sufficient to explain the variation in the odds of vaccination intention. Therefore, our finding on the collectivism-social influence interaction is inconclusive.

The lack of evidence of social influence that we found here has broader theoretical implications. Cultural evolution models make ample use of conformist transmission, and some conclude that conformism can promote prosocial behaviours like cooperation through cultural group selection (Boyd et al. 2011; Boyd \& Richerson 1982, 1985; Henrich \& Boyd 2001), while others argue that conformism can hinder them (Lehmann et al. 2008; Lehmann \& Feldman 2008; Molleman et al. 2013). Nevertheless, empirical studies testing these models are scarce. These models also largely ignore individual variation in susceptibility to social influence. A few experimental and empirical studies, however, have illustrated that there is much heterogeneity in social learning strategies (McElreath et al. 2008) and that not everyone is a conformist or a nonconformist (Efferson et al. 2008, 2015, 2020). Future theoretical models should therefore consider individual susceptibility to social influence when employing conformist transmission.

Previous findings have revealed a link between conservative political ideology and antivaccination attitudes (Hornsey 2020); however, our findings suggest that the direction of this link changes depending on the country. We previously demonstrated that vaccine hesitancy in Turkey decreases, and vaccine trust increases, from left- to right-wing political orientation (Salali \& Uysal 2021b). Here, the odds of vaccination intention among the unvaccinated in Turkey increased with a political orientation towards the right. Another study showed an increased likelihood of COVID-19 vaccination among the politically right wing in Mexico but a decreased likelihood among those in the US (Roozenbeek et al. 2020). The link between political orientation and vaccine hesitancy likely comes down to trust in government and specific institutions (Jamison et al. 2019). It is possible that governments' COVID-19 vaccine promotion campaigns and messages (simulated here) are taken more sympathetically among the right wing, who have greater trust in the government than the left wing. The lack of trust in the government among the unvaccinated left wing may contribute to their observed vaccination intention. Further studies should test the link between trust in government and vaccination decisions in Turkey.

\section{Policy implications}

Our findings suggest that advertising the percentage of those who have already received two doses of the COVID-19 vaccine in Turkey is not sufficient to induce a cascade of others getting themselves vaccinated. What is the take-home message for a policymaker, especially given the association of attitudes like reactance and 
collectivism with vaccination intention? There is clearly no "one-size-fits-all" strategy given the heterogeneity of attitudes associated with vaccine hesitancy. Policymakers should have a solid understanding of their society's traits, like collectivism, and deploy different strategies accordingly (Jarrett et al. 2015; Simas \& Larson 2021). In Turkey, the use of statistics on the percentage of double vaccinated may have no traction, but emphasizing the group benefit (i.e. "get your vaccine and protect your local community") may be a better nudge for vaccine-hesitant people who are more collectivistic. The same message, however, may not induce any change in reactant people. While some researchers suggest that vaccine mandates will induce more reactance and result in unsuccessful vaccine promotion (Schmelz \& Bowles 2021), others argue that the mandates will not necessarily backfire and can lead to greater compliance (Albarracin et al. 2021). Especially for highly reactant people, more creative and less controlling communication strategies emphasizing individual freedom should be considered (Hornsey 2020; Miller et al. 2007). With the right incentives, people may act beyond their self-interest (Chapman et al. 2012).

\section{Limitations and future directions}

Like most experimental studies conducted through the Internet, our sample had some limitations. For example, 520 out of 1013 participants had an undergraduate degree a relatively high percentage compared to country-specific education levels. Nevertheless, we controlled for education level, which did not emerge as a significant predictor of COVID-19 vaccination intention in our models. Furthermore, $65 \%$ of our participants came from the three most populated cities in Turkey. Therefore, the generalizability of our findings to under-represented cities and regions is not clear. Moreover, because our participation criterion was no prior doses of the COVID-19 vaccine, our sample could not be demographically representative. As such, there was a bias towards female participants (Table 1).

We also acknowledge that vaccination intention is not a perfect proxy for actual uptake. Future studies would benefit from a longitudinal design, where follow-up questions reveal whether unvaccinated people ultimately received the vaccine or not in the following months. Our study concerned the unvaccinated in Turkey; future studies should test whether conformist social influence affects the decision of the unvaccinated in other cultures.

\section{Conclusion}

In sum, our results suggest that conformist social influence does not change the odds of COVID-19 vaccination intention among unvaccinated people in Turkey. Instead, they offer new insight into the psychological factors that contribute to vaccination decisions. Understanding these factors facilitates the development of more effective vaccine promotion strategies. 
Acknowledgements. We thank Eva Brandl for her comments on an earlier version of this manuscript.

Ethics. This study was approved by the UCL Research Ethics Committee (ID: 13121/003).

Data accessibility. All data files and the $R$ code are available at https://osf.io/jb7zs/?view only=bfd3cb07ee3d478297bb55a9d19b659b

Authors' contributions. GDS and MSU conceived the project. All authors contributed to the experiment design. GDS and MSU did the statistical analyses. GDS wrote the paper and all authors commented on the drafts.

Financial support. This research was funded by University College London Global Challenges Research Fund. GDS holds a British Academy Postdoctoral Research Fellowship.

Competing interests. We declare we have no competing interests.

\section{References}

Albarracin, D., Jung, H., Song, W., Tan, A., \& Fishman, J. (2021). Rather than inducing psychological reactance, requiring vaccination strengthens intentions to vaccinate in US populations. Scientific Reports, 11(1), 1-9.

Arnot, M., Brandl, E., Campbell, O. L. K., ... Zhang, H. (2020). How evolutionary behavioural sciences can help us understand behaviour in a pandemic. Evolution, Medicine, and Public Health, 2020(1), 264-278.

Asch, S. E. (1956). Studies of independence and conformity: I. A minority of one against a unanimous majority. Psychological Monographs: General and Applied, 70(9), 1.

Bauch, C. T., \& Earn, D. J. D. (2004). Vaccination and the theory of games. Proceedings of the National Academy of Sciences of the United States of America, 101(36), 13391-13394.

Bond, R., \& Smith, P. B. (1996). Culture and conformity: A meta-analysis of studies using Asch's (1952b, 1956) line judgment task. Psychological Bulletin, 119(1), 111-137.

Boyd, R., \& Richerson, P. J. (1982). Cultural transmission and the evolution of cooperative behavior. Human Ecology, 10(3), 325-351.

Boyd, R., \& Richerson, P. J. (1985). Culture and the Evolutionary Process, Chicago: University of Chicago Press.

Boyd, R., Richerson, P. J., \& Henrich, J. (2011). Rapid cultural adaptation can facilitate the evolution of large-scale cooperation. Behavioral Ecology and Sociobiology, 65(3), 431-444.

Brehm, J. W. (1966). A theory of psychological reactance., New York: Academic press.

Chapman, G. B., Li, M., Vietri, J., ... Galvani, A. P. (2012). Using Game Theory to Examine Incentives in Influenza Vaccination Behavior. Psychological Science, 23(9), 1008-1015.

Efferson, C., Lalive, R., Richerson, P. J., McElreath, R., \& Lubell, M. (2008). 
Conformists and mavericks: the empirics of frequency-dependent cultural transmission. Evolution and Human Behavior, 29(1), 56-64.

Efferson, C., Vogt, S., Elhadi, A., Ahmed, E. H. F., \& Fehr, E. (2015). Female genital cutting is not a social coordination norm. Science, 349(6255).

Efferson, C., Vogt, S., \& Fehr, E. (2020). The promise and the peril of using social influence to reverse harmful traditions. Nature Human Behaviour, 4, 55-68.

Fu, F., Rosenbloom, D. I., Wang, L., \& Nowak, M. A. (2011). Imitation dynamics of vaccination behaviour on social networks. Proceedings of the Royal Society $B$ : Biological Sciences, 278(1702), 42-49.

Goldsmith, R. E., Clark, R. A., \& Lafferty, B. (2005). Tendency to conform: a new measure and its relationship to pyschological reactance. Pyscchologial Reportschologial Reports, 96, 591-594.

Hardin, G. (1968). Tragedy of the Commons. Science, 162, 1243-1248.

Henrich, J., \& Boyd, R. (1998). The evolution of conformist transmission and the emergence of between-group differences. Evolution and Human Behavior, 19(4), 215-241.

Henrich, J., \& Boyd, R. (2001). Why people punish defectors. Weak conformist transmission can stabilize costly enforcement of norms in cooperative dilemmas. Journal of Theoretical Biology, 208(1), 79-89.

Hofstede, G. (2001). Culture's consequences: Comparing values, behaviors, institutions and organizations across nations, Sage publications.

Hong, S., \& Faedda, S. (1996). Refinement of the Hong psychological reactance scale. Educational and Pyschological Measurement, 56(1), 173-182.

Hornsey, M. J. (2020). Why Facts Are Not Enough: Understanding and Managing the Motivated Rejection of Science. Current Directions in Psychological Science, 29(6), 583-591.

Hornsey, M. J., Harris, E. A., \& Fielding, K. S. (2018). The psychological roots of anti-vaccination attitudes: A 24-nation investigation. Health Psychology, 37(4), 307-315.

Howard, J. A., \& Gibson, M. A. (2017). Frequency-dependent female genital cutting behaviour confers evolutionary fitness benefits. Nature Ecology and Evolution, 1(3), 1-6.

Ibuka, Y., Li, M., Vietri, J., Chapman, G. B., \& Galvani, A. P. (2014). Free-riding behavior in vaccination decisions: An experimental study. PLOS ONE, 9(1), 1-9. Imhoff, R., \& Erb, H. P. (2009). What motivates nonconformity? Uniqueness seeking blocks majority influence. Personality and Social Psychology Bulletin, 35(3), 309-320.

Imhoff, R., \& Lamberty, P. K. (2017). Too special to be duped: Need for uniqueness motivates conspiracy beliefs. European Journal of Social Psychology, 47(6), 724-734.

Jamison, A. M., Quinn, S. C., \& Freimuth, V. S. (2019). “You don't trust a government vaccine": Narratives of institutional trust and influenza vaccination among African American and white adults. Social Science and Medicine, 221, 87-94. 
Jansen, V. A. A., Stollenwerk, N., Jensen, H. J., Ramsay, M. E., Edmunds, W. J., \& Rhodes, C. J. (2003). Measles outbreaks in a population with declining vaccine uptake. Science, 301(5634), 804.

Jarrett, C., Wilson, R., O'Leary, M., ... Schuster, M. (2015). Strategies for addressing vaccine hesitancy - A systematic review. Vaccine, 33(34), 4180-4190.

Lehmann, L., \& Feldman, M. W. (2008). The co-evolution of culturally inherited altruistic helping and cultural transmission under random group formation. Theoretical Population Biology, 73(4), 506-516.

Lehmann, L., Feldman, M. W., \& Foster, K. R. (2008). Cultural transmission can inhibit the evolution of altruistic helping. The American Naturalist, 172(1), 12-24.

Lu, J. G., Jin, P., \& English, A. S. (2021). Collectivism predicts mask use during COVID-19. Proceedings of the National Academy of Sciences of the United States of America, 118(23). doi:10.1073/pnas.2021793118

McElreath, R., Bell, A. V., Efferson, C., Lubell, M., Richerson, P. J., \& Waring, T. (2008). Beyond existence and aiming outside the laboratory: Estimating frequency-dependent and pay-off-biased social learning strategies.

Philosophical Transactions of the Royal Society B: Biological Sciences, 363(1509), 3515-3528.

Miller, C. H., Lane, L. T., Deatrick, L. M., Young, A. M., \& Potts, K. A. (2007). Psychological reactance and promotional health messages: The effects of controlling language, lexical concreteness, and the restoration of freedom. Human Communication Research, 33(2), 219-240.

Molleman, L., Pen, I., \& Weissing, F. (2013). Effects of Conformism on the Cultural Evolution of Social Behaviour. PloS One, 8(7). doi:10.1371/journal.pone.0068153

Muthukrishna, M., \& Schaller, M. (2020). Are Collectivistic Cultures More Prone to Rapid Transformation? Computational Models of Cross-Cultural Differences, Social Network Structure, Dynamic Social Influence, and Cultural Change. Personality and Social Psychology Review, 24(2), 103-120.

Roozenbeek, J., Schneider, C. R., Dryhurst, S., ... Van Der Linden, S. (2020). Susceptibility to misinformation about COVID-19 around the world. Royal Society Open Science, 7(10). doi:10.1098/rsos.201199

Salali, G. D., \& Uysal, M. S. (2020). COVID-19 vaccine hesitancy is associated with beliefs on the origin of the novel coronavirus in the UK and Turkey.

Psychological Medicine, 1-3.

Salali, G. D., \& Uysal, M. S. (2021a). Effective incentives for increasing COVID-19 vaccine uptake. Psychological Medicine, 1-3.

Salali, G. D., \& Uysal, M. S. (2021b). Why Some Hesitate More: Cross-Cultural Variation in Conspiracy Beliefs, Belief in Science and Vaccine Attitudes. MedRxiv. doi:https://doi.org/10.1101/2021.07.09.21260228

Schmelz, K., \& Bowles, S. (2021). Overcoming COVID-19 vaccination resistance when alternative policies affect the dynamics of conformism, social norms, and crowding out. Proceedings of the National Academy of Sciences of the United States of America, 118(25), 1-7. 
Simas, C., \& Larson, H. J. (2021). Overcoming vaccine hesitancy in low-income and middle-income regions. Nature Reviews Disease Primers, 7(1), 1-2.

Taylor, S., \& Asmundson, G. J. G. (2021). Negative attitudes about facemasks during the COVID-19 pandemic: The dual importance of perceived ineffectiveness and psychological reactance. PLOS ONE, 16(2 February), 1-15. WHO. (2020). Measles and Rubella Surveillance Data. Retrieved July 18, 2020, from https://www.who.int/immunization/monitoring_surveillance/burden/vpd/surveillan ce_type/active/measles_monthlydata/en/ 